\title{
The impact of postnatal antibiotics on the preterm intestinal microbiome
}

\author{
Majd Dardas' ${ }^{1}$ Steven R. Gill ${ }^{2,3}$, Alex Grier ${ }^{3}$, Gloria S. Pryhuber' ${ }^{1}$ Ann L. Gill'², Yi-Horng Lee ${ }^{4}$ and Ronnie Guillet ${ }^{1}$
}

BACKGROUND: Development of the intestinal microbiome in preterm infants has significant impact on infant health. Our objective was to determine if duration of antibiotics within the first 10 and $30 \mathrm{~d}$ after birth affects the intestinal microbiome.

METHODS: Subjects were 24 0/7-31 6/7 wk of gestational age who received $\geq 50 \%$ breast milk and a total of $\geq 100 \mathrm{ml} / \mathrm{kg}$ of feeds by $10 \mathrm{~d}$. Rectal (fecal) swabs were collected at 10 and $30 \mathrm{~d}$ and analyzed by 165 rRNA pyrosequencing. At both time points, we examined the rectal microbiome from infants who received only $2 \mathrm{~d}$ of antibiotics and those who received at least $7 \mathrm{~d}$ of antibiotics.

RESULTS: In the 29 infants enrolled in our study, we found a decrease in diversity index from $10 \mathrm{~d}$ samples in those who received more antibiotics. Such difference in diversity and richness was not as pronounced in $30 \mathrm{~d}$ samples. Firmicutes and Bacteroidetes were most abundant in the $10 \mathrm{~d}$ samples. While these two phyla remained dominant in $30 \mathrm{~d}$ samples, there was an increase in Proteobacteria and Actinobacteria.

CONCLUSION: Despite antibiotic therapy, neonates continued to acquire bacteria in the gastrointestinal tract. The process of bacterial acquisition is perturbed with the use of antibiotics.

T he process of acquiring bacteria in the neonatal gastrointestinal tract can play a significant role in the development of the immune system that may influence the infant's future health (1). This process has been linked to the pathogenesis of necrotizing enterocolitis (NEC) $(2,3)$, a devastating intestinal disease affecting primarily premature infants. Although the exact association and mechanisms between neonatal intestinal microbiota and NEC remain unclear, the interplay between them appears to be a dynamic one. As early as 2004, a temporal association between early colonization with Clostridium and later development of NEC was suggested (4). This temporal relationship is again illustrated by the observation of a differing intestinal microbiome $1 \mathrm{wk}$ prior to the onset of NEC (5). These reports point to the fact that both the bacterial composition of the gastrointestinal tract and its colonization process may be important in the pathogenesis of NEC.

Clinical practices such as the use of $\mathrm{H}_{2}$ blockers and antibiotics are associated with increased incidence of NEC $(6,7)$.
Feeding with maternal breast milk (8), donor breast milk, (9) and probiotics (10-12) have been shown to decrease such risk. While the mechanisms of how these clinical practices impact the pathogenesis of NEC are not clearly understood, it is conceivable that they all influence the modulation of the bacterial colonization process in the nascent gastrointestinal tract. We have chosen to focus our study on the impact of antibiotic therapy on the development of intestinal microbiota in the neonatal gastrointestinal tract. We hypothesized that neonatal gastrointestinal tract colonization pattern is influenced by the duration of antibiotic therapy and that the diversity of the intestinal microbiome of premature infants inversely correlates with the duration of exposure to antibiotics.

\section{RESULTS}

The patient recruitment schema is illustrated in Figure 1. We screened 108 infants born at gestational ages less than $32 \mathrm{wk}$. Forty-four infants met inclusion criteria, and consents were obtained for 29 infants to enroll in the study. Of the 29 infants enrolled, 27 received at least $50 \%$ of their feeds as maternal breast milk and 2 received exclusively formula. Samples from these two exclusively formula-fed infants were excluded from analysis in order to ensure a more homogeneous population in terms of feeding material.

There were $1510 \mathrm{~d}$ samples collected from infants who received only $2 \mathrm{~d}$ of antibiotics, and $1210 \mathrm{~d}$ samples were collected from infants who received the same antibiotics for 7-10 d. Antibiotics administered in the first $10 \mathrm{~d}$ of life were limited to ampicillin and gentamicin. The $30 \mathrm{~d}$ samples were collected from 21 out of 27 of the infants for whom we had $10 \mathrm{~d}$ samples. We could not collect six $30 \mathrm{~d}$ samples because these infants were transferred to other facilities for convalescent care. The $30 \mathrm{~d}$ samples were segregated into two groups based on the total duration of antibiotics received, minimal exposure ( $2 \mathrm{~d}$ ) and prolonged exposure ( $\geq 7 \mathrm{~d})$. There was one patient in the 2-d antibiotic group at age of $10 \mathrm{~d}$ who subsequently received more than $5 \mathrm{~d}$ of antibiotics, and the $30 \mathrm{~d}$ sample from this patient was crossed over to the group who have received $\geq 7 \mathrm{~d}$ of antibiotics. There were two patients from the 2-d antibiotic group at age 10 received 2 additional days of antibiotic therapy; hence, they did not fit in the criterion of

'Division of Neonatology, Department of Pediatrics, University of Rochester Medical Center, Rochester, New York; ${ }^{2}$ Department of Microbiology and Immunology, University of Rochester Medical Center, Rochester, New York; ${ }^{3}$ Genomics Research Center, University of Rochester Medical Center, Rochester, New York; ${ }^{4}$ Division of Pediatric Surgery, Department of Surgery, University of Rochester Medical Center, Rochester, New York. Correspondence: Ronnie Guillet (Ronnie_Guillet@URMC.Rochester.edu) 


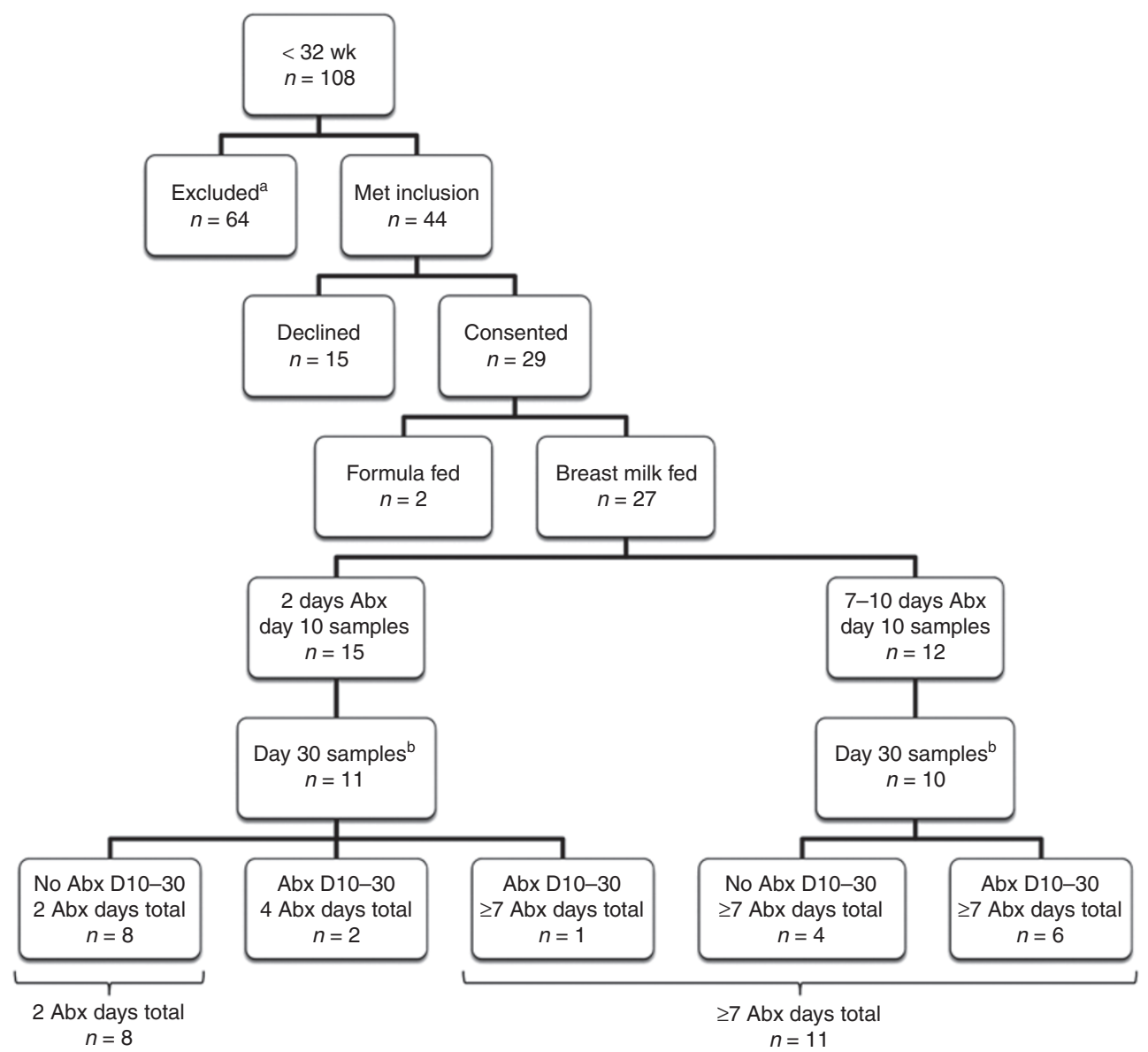

Figure 1. Patient cohort used for the preterm intestinal microbiome study. One-hundred eight infants $<32$ wk of age were selected for the study. Of these, 27 infants met the inclusion criteria, received $\geq 50 \%$ breast milk, and received multiple courses of antibiotic therapy (Abx). alnfants who remained NPO for $\geq 7 \mathrm{~d}$, who had necrotizing enterocolitis, intestinal perforation, or any other gastrointestinal anomaly within the first $10 \mathrm{~d}$ of life, or those whose

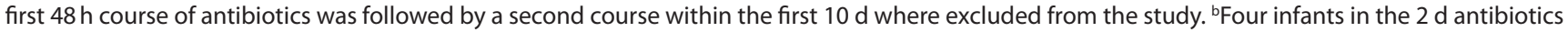
group and two infants in the 7-10 d group were transferred to another facility and thus no samples could be obtained.

receiving only $2 \mathrm{~d}$ or $\geq 7 \mathrm{~d}$ of antibiotic therapy, and their 30 $\mathrm{d}$ samples were excluded from analysis. Patients who received only $2 \mathrm{~d}$ of antibiotic therapy were treated with ampicillin and gentamicin. Patients who receive additional antibiotic therapy may have received ampicillin, gentamicin, vancomycin, cefazolin, cefotaxime, and/or erythromycin.

Infant and maternal demographics were similar in both groups except that there were more infants in the group receiving more antibiotics born by C-section (Table 1 ). Infants who received $2 \mathrm{~d}$ of antibiotics $(n=15)$ and those who received $7-10 \mathrm{~d}$ of antibiotics $(n=12)$ by $10 \mathrm{~d}$ of age were similar in terms of their weights, total volume of feeds $/ \mathrm{kg}$, number of days with at least one feed, and percentage breast milk of total feeds (Table 2). The $30 \mathrm{~d}$ samples were segregated based on the number of days of antibiotic therapy. Infants who received only $2 \mathrm{~d}$ of antibiotics $(n=8)$ and those who received at least $7 \mathrm{~d}$ of antibiotics $(n=11)$ were similar in terms of their weights and percentage breast milk of total feeds at day 30 but were significantly different in terms of total volume of feeds/ $/ \mathrm{kg}$ and number of days with at least one feed (Table 3). This difference is expected since the infants who receive more antibiotics were more likely to be given nothing by mouth for concern of sepsis. None of these patients had culture-positive sepsis, but were placed on empiric antibiotic therapy because of clinical concerns. None of the infants in our study received proton pump inhibitors or $\mathrm{H} 2$ blockers before day 30 of age.

Despite these clinical similarities, we found a significantly lower Shannon diversity index from $10 \mathrm{~d}$ samples in infants who received antibiotics for $7-10 \mathrm{~d}$ as compared to those who received only $2 \mathrm{~d}$ after adjusting for mode of delivery as a confounder. There was also a decrease in richness, but the difference is not statistically significant (Table 2). While the bacterial phyla present in the two groups are relatively similar, there was a significant difference in their relative abundance. Such differences in richness and diversity were no longer observed in $30 \mathrm{~d}$ samples (Table 3).

In evaluating each patient's rectal microbiota within each sample group, we found a subset of 10 and $30 \mathrm{~d}$ samples with distinct compositions at the phylum level that were vastly different from the remainder of the group (Figure 2). Interestingly, the patients with atypical composition in their $10 \mathrm{~d}$ samples were not the same as those of $30 \mathrm{~d}$ samples, and there were no distinct features identified in these patients' demographic information. In the $10 \mathrm{~d}$ samples from patients who received 
Table 1. Infant and maternal demographics of breast milk-fed infants

\begin{tabular}{|c|c|c|c|}
\hline & $\begin{array}{c}2 \mathrm{~d} \text { of antibiotics } \\
n=15\end{array}$ & $\begin{array}{c}7-10 \mathrm{~d} \text { of } \\
\text { antibiotics } n=12\end{array}$ & $P$ value \\
\hline $\begin{array}{l}\text { Gender } \\
\text { (male/female) }\end{array}$ & $6 / 9$ & $6 / 6$ & * \\
\hline $\begin{array}{l}\text { Gestational age } \\
\text { at birth (wks) } \\
\text { (mean } \pm \text { SD) }\end{array}$ & $29.2 \pm 1.7$ & $28.5 \pm 2.4$ & * \\
\hline $\begin{array}{l}\text { Birth weight (g) } \\
\text { (mean } \pm S D)\end{array}$ & $1339 \pm 328$ & $1147 \pm 401$ & * \\
\hline Race (white/black) & $11 / 3$ & $6 / 1$ & * \\
\hline $\begin{array}{l}\text { Ethnicity } \\
\text { (Hispanic or Latino) }\end{array}$ & $2 / 15$ & $2 / 12$ & * \\
\hline $\begin{array}{l}\text { Delivery } \\
\text { (vaginal/C-section) }\end{array}$ & $13 / 2$ & $2 / 10$ & $* *$ \\
\hline $\begin{array}{l}\text { APGAR score at } 1 \mathrm{~min} \\
\text { (median) }\end{array}$ & 5 & 2 & * \\
\hline $\begin{array}{l}\text { APGAR score at } 5 \mathrm{~min} \\
\text { (median) }\end{array}$ & 7 & 6 & * \\
\hline $\begin{array}{l}\text { Mechanical } \\
\text { ventilator }\end{array}$ & $8 / 15$ & $11 / 12$ & * \\
\hline $\begin{array}{l}\text { Number of days on } \\
\text { ventilator } \\
\text { (mean } \pm \text { SD) }\end{array}$ & $2.7 \pm 7.0$ & $7.4 \pm 11.5$ & * \\
\hline $\begin{array}{l}\text { Maternal age (years) } \\
(\text { mean } \pm \text { SD) }\end{array}$ & $26.6 \pm 6.3$ & $29.1 \pm 5.4$ & * \\
\hline $\begin{array}{l}\text { Maternal group B } \\
\text { Strep positive }\end{array}$ & $4 / 12$ & $3 / 9$ & * \\
\hline $\begin{array}{l}\text { Prolonged rupture of } \\
\text { membranes }>18 \mathrm{~h}\end{array}$ & $5 / 15$ & $4 / 12$ & * \\
\hline $\begin{array}{l}\text { Chorioamnionitis } \\
\text { (placental pathology) }\end{array}$ & $6 / 15$ & $5 / 11$ & * \\
\hline $\begin{array}{l}\text { Chorioamnionitis } \\
\text { (clinical diagnosis) }\end{array}$ & $1 / 15$ & $1 / 12$ & * \\
\hline Maternal antibiotics & $12 / 15$ & $6 / 12$ & * \\
\hline Gestational diabetes & $0 / 15$ & $3 / 12$ & * \\
\hline Pre-eclampsia & $3 / 15$ & $0 / 12$ & * \\
\hline
\end{tabular}

Patient characteristics were similar in both groups except that infants who received more antibiotics were significantly more likely to be born via C-section.

${ }^{*} P$ value not statistically significant. ${ }^{* *} P$ value $<0.05$. only $2 \mathrm{~d}$ of antibiotic therapy, the majority of samples contained Firmicutes as their predominant phylum while the patients with atypical composition predominantly had Bacteroidetes regardless of the duration of antibiotics exposure. In the $30 \mathrm{~d}$ samples, the predominant phylum remained Firmicutes, but there was a relative rise in Actinobacteria and Proteobacteria. The bacterial composition of all samples on the genus level is illustrated in Figure 3. The number of genera present in each sample is more balanced in $30 \mathrm{~d}$ samples compared to $10 \mathrm{~d}$ samples in both 2-d antibiotic treatment and $\geq 7 \mathrm{~d}$ antibiotic treatment groups. Samples from patients receiving less antibiotic therapy have a more even distribution of various genera.

We segregated those with atypical composition from the remainder of the group in our calculation of Shannon diversity index. The increase in diversity over time and the decrease in diversity with additional use of antibiotic therapy are illustrated in Figure 4. The difference between groups is represented by $\beta$-diversity as expressed in Bray-Curtis index. Overall, the $30 \mathrm{~d}$ samples are more dissimilar to other groups comparing to the $10 \mathrm{~d}$ samples (Figure 5a). The greatest difference is seen between $10 \mathrm{~d}$ samples from patients who received $\geq 7 \mathrm{~d}$ of antibiotics and $30 \mathrm{~d}$ samples from patients who have received only $2 \mathrm{~d}$ of antibiotics. The least difference is observed between $10 \mathrm{~d}$ sample from those who received only $2 \mathrm{~d}$ of antibiotics and those who received antibiotics for $\geq 7 \mathrm{~d}$. The difference in $\beta$-diversity index between atypical and typical composition within the 2- $\mathrm{d}$ antibiotic group and $\geq 7-\mathrm{d}$ antibiotic group from both time points are greater than that between the groups (Figure 5b).

\section{DISCUSSION}

Our data suggest that the neonatal intestinal microbiome can be affected by both chronologic age and antibiotic exposure. Rectal microbiota diversity increases over time but decreases with antibiotic exposure. Among infants receiving the majority of their feeds as maternal breast milk and after adjusting for mode of delivery as a confounder, we found a statistically significant decrease in diversity at $10 \mathrm{~d}$ in infants who received antibiotics for 7-10 d compared to those who received $2 \mathrm{~d}$ of

Table 2. Characteristics and outcomes of breast milk-fed infants at age $10 \mathrm{~d}$

\begin{tabular}{|c|c|c|c|c|}
\hline & $2 \mathrm{~d}$ antibiotics $n=15$ & $7-10 \mathrm{~d}$ antibiotics $n=12$ & $P$ value & Adjusted $P$ value \\
\hline Weight at day $10(\mathrm{~g})($ mean \pm SD) & $1,367 \pm 341$ & $1,140 \pm 384$ & * & \\
\hline Day of first feed (mean \pm SD) & $2.0 \pm 1.0$ & $2.6 \pm 1.2$ & * & \\
\hline Number of days with $\geq 1$ feed (mean \pm SD) & $8.4 \pm 1.1$ & $7.8 \pm 1.7$ & * & \\
\hline$\%$ Breast milk of total feeds (mean \pm SD) & $98.4 \pm 3.0$ & $96.2 \pm 4.9$ & * & \\
\hline Sample collection day (mean \pm SD) & $10.5 \pm 0.7$ & $10.8 \pm 0.4$ & * & \\
\hline Shannon's diversity index (mean \pm SD) & $0.8 \pm 0.4$ & $0.6 \pm 0.4$ & * & ** \\
\hline Richness (mean $\pm \mathrm{SD}$ ) & $13.6 \pm 7.2$ & $12.7 \pm 5.6$ & * & * \\
\hline
\end{tabular}

For breast milk fed infants and after adjusting for mode of delivery as a confounder, there was a significant decrease in diversity at $10 \mathrm{~d}$ in infants who received antibiotics for $7-10 \mathrm{~d}$ compared to those who received $2 \mathrm{~d}$.

${ }^{*}$ v value not statistically significant. ${ }^{* *} P$ value $<0.05$. 
Table 3. Characteristics and outcomes of breast milk-fed infants at age $30 \mathrm{~d}$

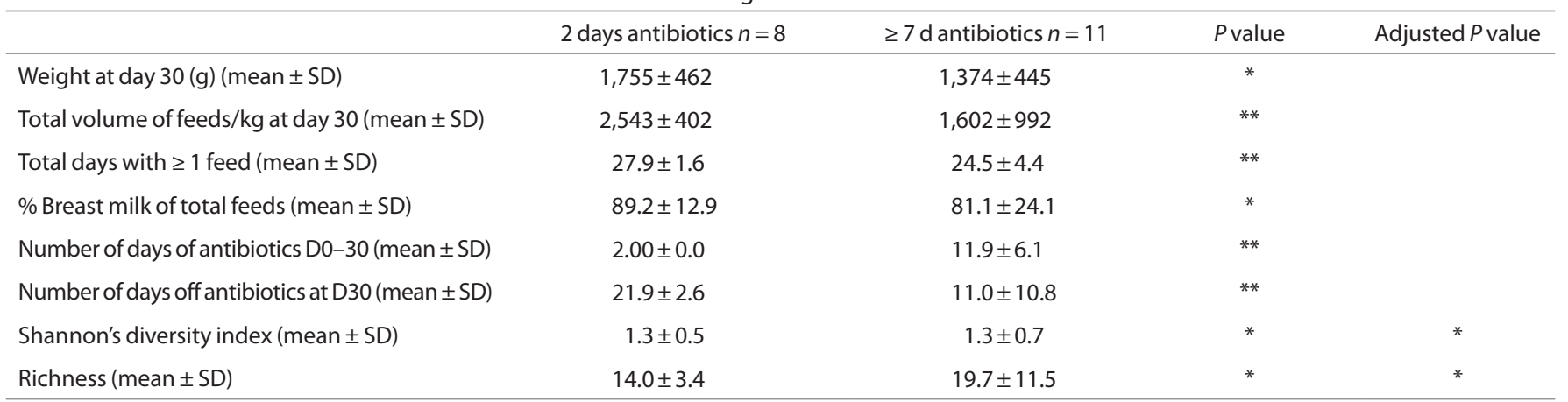

Two infants were excluded from the analysis because they received $4 \mathrm{~d}$ of antibiotics. For breast milk-fed infants and after adjusting for mode of delivery as a covariate, infants exposed to more days of antibiotics had higher Bacteroidetes proportion at day of life 10. This achieved statistical significance.

${ }^{*} P$ value not statistically significant. ${ }^{*} P$ value $<0.05$.

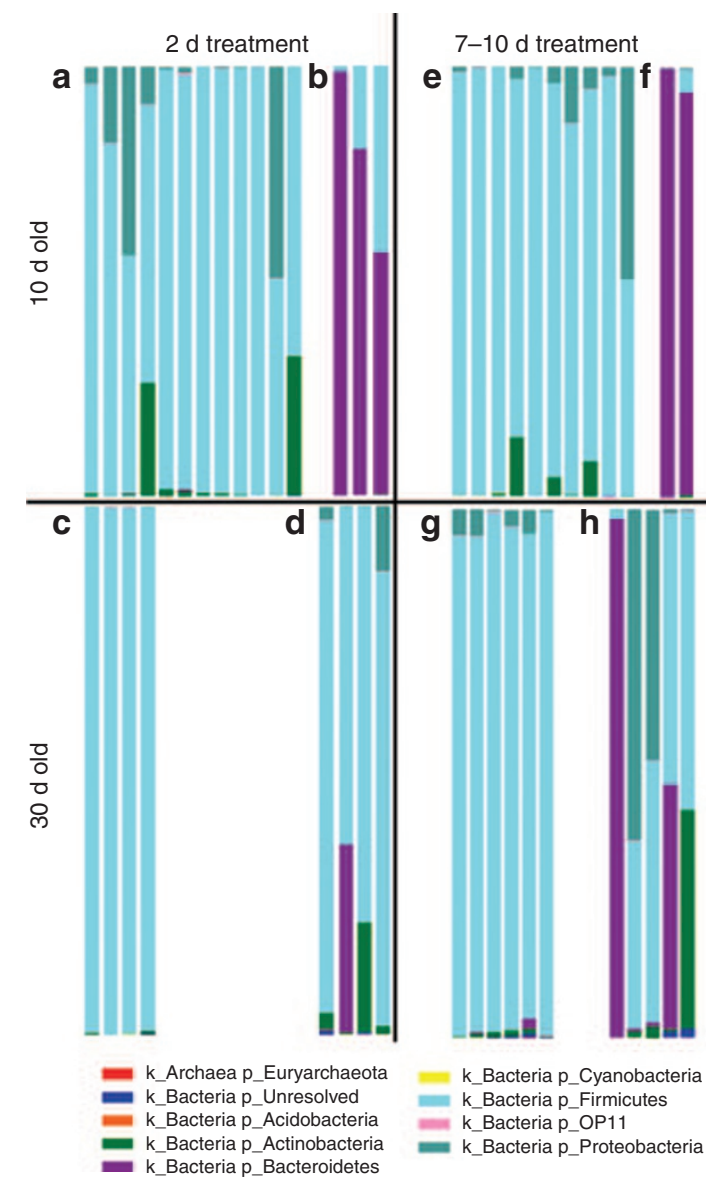

Figure 2. Phylum level composition of day 10 and day 30 samples. Bar plots of phyla relative abundance observed in 27 infants at 10 and $30 \mathrm{~d}$ of age and after $2 \mathrm{~d}$ and 7-10 d of antibiotic treatment. a, c, e, and $\mathbf{g}$ represent typical subgroups. b, $\mathbf{d}, \mathbf{f}$, and $\mathbf{h}$ represent atypical subgroups. Color codes for phyla represented in the bar plots are shown below the figure.

antibiotics. In addition, by day 30 , we noted a shift in the number of genera comprising the microbiome. The difference in the Shannon diversity index observed at $10 \mathrm{~d}$ of age was no longer seen by $30 \mathrm{~d}$ of age. This suggests that the neonatal gastrointestinal tract continues to acquire different bacterial genera despite antibiotic pressure. While the earlier antibiotic therapy is more uniform (i.e., ampicillin and gentamicin) and tends to be broad-spectrum, later antibiotic agents are more focused in their antimicrobial targets because patient condition is better known, source of infection is more clear, and a wider variety of agents have been used. Different antimicrobial agents may exert differential impact on the intestinal microbiota. Our study does not address the timing of recovery in microbiota after antibiotic therapy has been discontinued. These factors may contribute to the relative similarities between the two groups of $30 \mathrm{~d}$ samples.

We are not able to identify any factors that may explain how certain individuals acquire atypical bacterial composition that are vastly different from others within the same group in both 10 and $30 \mathrm{~d}$ samples. Again, we must be clear that the outliers from $10 \mathrm{~d}$ are not the same as those from $30 \mathrm{~d}$. As always with human subjects, there are variables beyond the scope of our data collection. This makes a strong argument that our intestinal microbiome is far more apt to be influenced by clinical intervention and environmental factors than genetic background. This is consistent with previous observations that life events such as changing dietary content, onset of illness, and use of antibiotics can alter our microbiota (13).

While Firmicutes and Bacteroidetes dominated the $10 \mathrm{~d}$ samples, there is a rise in the Proteobacteria and Actinobacteria in $30 \mathrm{~d}$ samples with corresponding decrease in Firmicutes and Bacteroidetes. The change is more pronounced in patients who have received more antibiotic therapy. A similar increase in Proteobacteria and decrease in the proportion of Firmicutes was observed by Mai et al. (5) in NEC infants over the week prior to the diagnosis of NEC. On a genus level, $10 \mathrm{~d}$ samples with only $2 \mathrm{~d}$ of antibiotic treatment have the greatest abundance of Lactococcus in the typical composition subgroup and Bacteroides in the atypical subgroup. The $30 \mathrm{~d}$ typical subgroup shows an increase in Clostridium and Anaerococcus in the atypical composition subgroup. With chronological progression, not only do the diversity of rectal microbiota increase as evidenced by Shannon diversity index, each group has become more dissimilar from one another as seen by increased BrayCurtis dissimilarity index.

The relationship between NEC and the changes in microbiota is a controversial one. Some studies have shown that 


\section{Articles $\mid$ Dardas et al.}

a

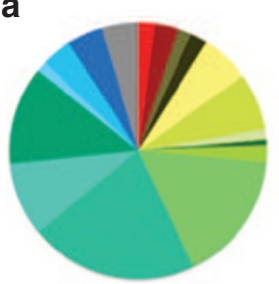

b

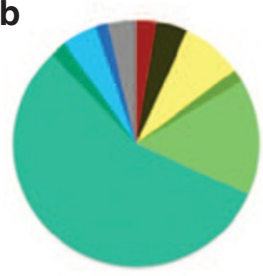

c
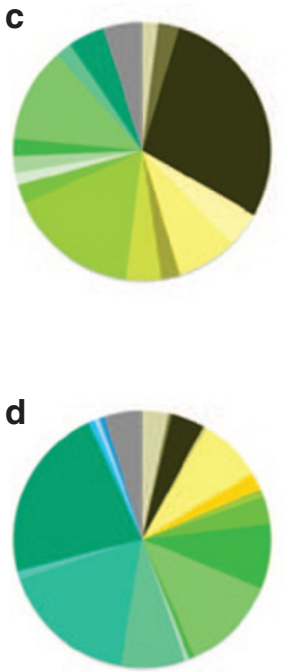

e

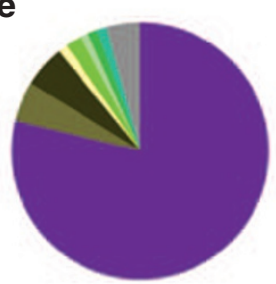

f

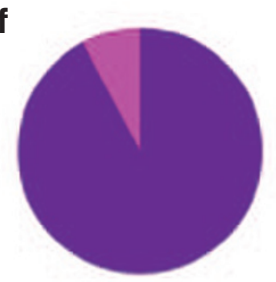

g

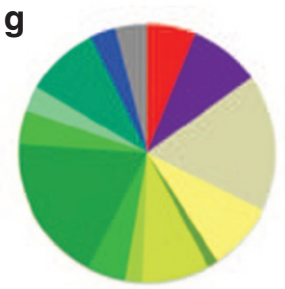

h

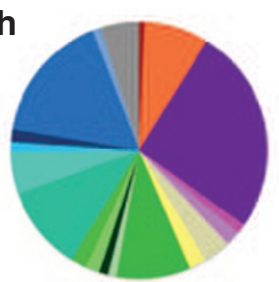

- Actinobacteria Actinomyces

- Actinobacteria Corynebacterium

- Actinobacteria Propionibacterium

- Bacteroidetes Bacteroides

- Bacteroidetes Parabacteroides

n Bacteroidetes Prevotella

Firmicutes Anaerococcus

- Firmicutes Blautia

- Firmicutes Clostridium

Firmicutes Coprococcus

nirmicutes Dialister

Firmicutes Enterococcus

Firmicutes Eubacterium

= Firmicutes f_clostridiaceae g_02d06

- Firmicutes f_clostridiaceae g_other

= Firmicutes f_clostridiaceae g_unresolved

ar Firmicutes f_erysipelotrichaceae g_unresolved

= Firmicutes f_lachnospiraceae g_other

n Firmicutes f_lachnospiraceae g_unresolved

= Firmicutes f_peptostreptococcaceae g_unresolved

= Firmicutes f_ruminococcaceae g_unresolved

= Firmicutes Faecalibacterium

n Firmicutes Finegoldia

- Firmicutes lactobacillales f_unresolved

Firmicutes Lactobacillus

= Firmicutes Lactococcus

- Firmicutes Leuconostoc

- Firmicutes o_clostridiales f_other

= Firmicutes o_clostridiales f_unresolved

- Firmicutes o_lactobacillales f_unresolved

- Firmicutes Peptoniphilus

n Firmicutes Peptostreptococcus

Inirmicutes Roseburia

n Firmicutes Ruminococcus

- Firmicutes Staphylococcus

nirmicutes Streptococcus

- Firmicutes Veillonella

= Proteobacteria f_enterobacteriaceae f_other

[ Proteobacteria f_enterobacteriaceae g_unresolved

- Proteobacteria f_hyphomicrobiaceae g_unresolved

- Proteobacteria f_rhodobacteraceae g_unresolved

- Proteobacteria Marinicella

- Proteobacteria o_burkholderiales f_unresolved

- Proteobacteria Serratia

- Proteobacteria Sutterella

- All else

Figure 3. Mean abundances of constituent genera of day 10 and day 30 samples. Pie charts of genera relative abundance observed in 27 infants at 10 and $30 \mathrm{~d}$ of age and after $2 \mathrm{~d}$ and $7-10 \mathrm{~d}$ of antibiotic treatment. Each panel represents typical or atypical subgroups, age, and days of treatment. Panel a: typical subgroup, $10 \mathrm{~d}$ old, $2 \mathrm{~d}$ of treatment. Panel b: typical subgroup, $10 \mathrm{~d}$ old, $7 \mathrm{~d}$ of treatment. Panel c: typical subgroup, $30 \mathrm{~d}$ old, $2 \mathrm{~d}$ of treatment. Panel d: typical subgroup, $30 \mathrm{~d}$ old, $7 \mathrm{~d}$ of treatment. Panel e: atypical subgroup, $10 \mathrm{~d}$ old, $2 \mathrm{~d}$ of treatment. Panel f: atypical subgroup, $10 \mathrm{~d}$ old, $7 \mathrm{~d}$ of treatment. Panel g: atypical subgroup, $30 \mathrm{~d}$ old, $2 \mathrm{~d}$ of treatment. Panel $\mathbf{h}$ : atypical subgroup, $30 \mathrm{~d}$ old, $7 \mathrm{~d}$ of treatment. Color codes for phyla represented in the bar plots are shown to the right of the figure.

infants who developed NEC had lower Firmicutes and higher Proteobacteria in their intestinal microbiome $(2,5)$, but others claim that there is no difference in the composition of intestinal microbiota in those who develop NEC (14). Wang et al. (2) have reported the most striking difference in intestinal microbiota between NEC and non-NEC infants, but fecal samples were obtained after the diagnosis of NEC had been made. The control samples were taken from non-NEC infants born at the same gestational age and collected on the match day of NEC diagnosis. There is no description regarding the temporal relationship to any antibiotic therapy either prior to NEC or related to NEC. Mai et al. (5) have reported some dissimilarity between NEC and non-NEC infants' intestinal microbiota from their fecal samples obtained $1 \mathrm{wk}$ prior to the onset of NEC, but not at a time closer to the diagnosis of NEC. There were mention of antibiotic duration for these infants, but no specific analysis has been done with respect to the timing, duration, or the types of antibiotics used. Although our study did not specifically address the difference in intestinal microbiome with respect to the onset of NEC, we speculate that our findings of altered intestinal microbiome in infants exposed to more antibiotics could be a factor that contributes to the understanding of how antibiotics are implicated in the pathogenesis of NEC.

Our study targeted preterm infants who were at highest risk of developing NEC. This particular population has 


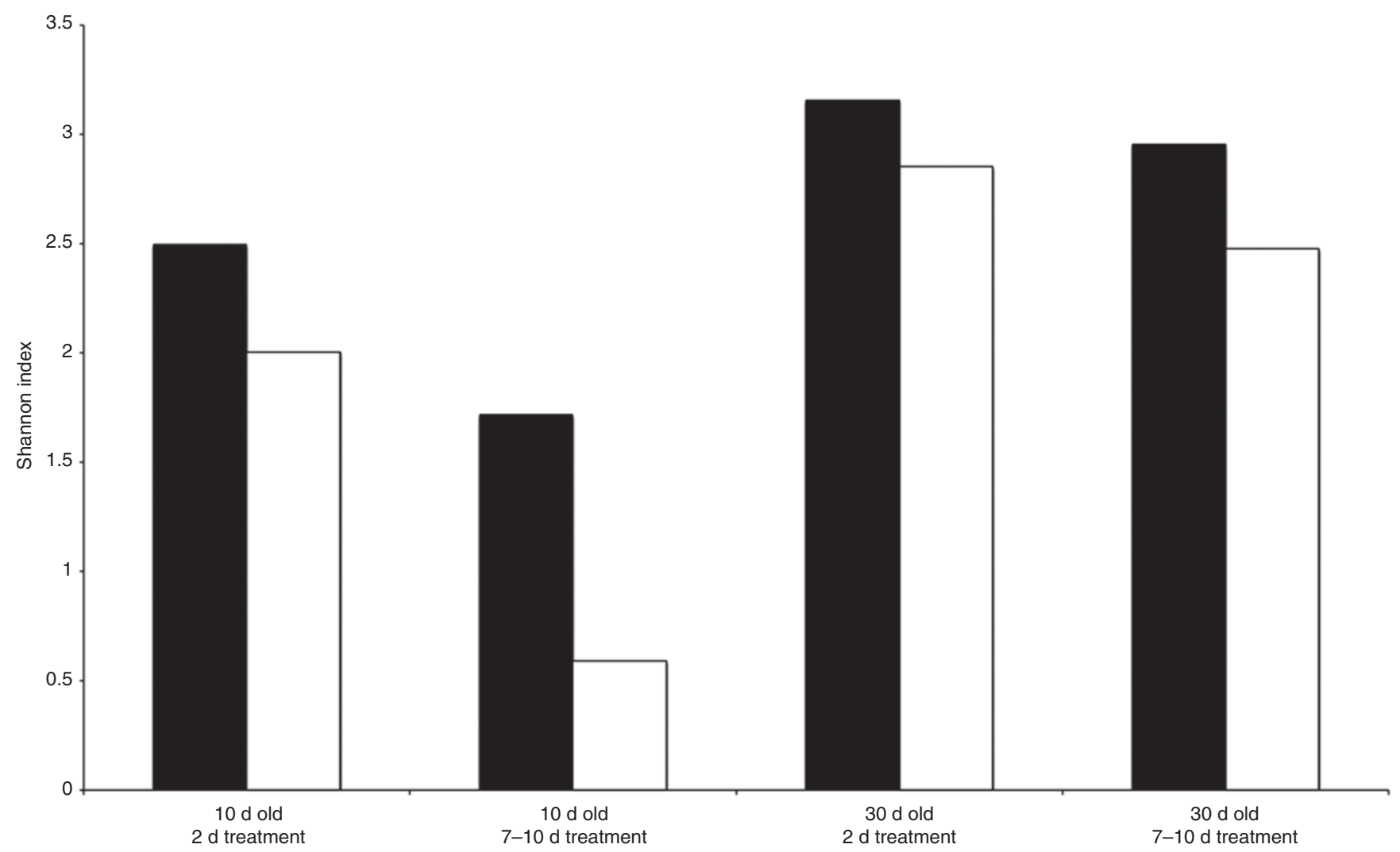

Figure 4. Shannon diversity index ( $\alpha$-diversity) based on age and antibiotic treatment. Diversity within each of the four groups separated by age, antibiotic treatment, and typical vs. atypical composition was determined using Shannon's diversity index. Samples with typical composition are shown in black bars. Samples with atypical composition are shown in white bars.

generally been underrepresented in many of the prior microbiome studies that were focused on late to full-term infants. The cohort in our study was relatively homogeneous in their feeding material, primarily maternal breast milk. This enables a more accurate depiction of the effect of antibiotics on the microbiome without the confounding variable of feeding content. It would have been interesting to compare the difference in microbiota between those who receive maternal breast milk vs. formula, but our success in advocating for maternal breast milk has made this question impossible to answer in our current study. The 27 breast milk-fed babies received $87.4 \%$ breast milk by volume at day 10 and the remaining 21 babies after transfers received $82 \%$ breast milk by volume at day 30 .

Choosing infants who have fed at least $100 \mathrm{ml} / \mathrm{kg}$ total feeds over the first $10 \mathrm{~d}$ of life may have selected against the sicker and more premature infants who could be at higher risk of NEC. Future studies comparing feeding infants with those kept NPO will improve our understanding of how feeds influence the establishment of the microbiome. Although we did not have a "true control" group with no antibiotics exposure due to the fact that all preterm infants in the studies received at least $2 \mathrm{~d}$ of empiric antibiotics at birth for presumed sepsis, we were able to demonstrate that different duration of antibiotics have different effects on the microbiome in terms of diversity and composition. Further studies with controls having no antibiotics exposure will help more accurately explain the natural course of microbiome establishment in preterm infants.

We noted that the infants born by $\mathrm{C}$-sections were more likely to receive more antibiotics. In many instances, babies who are born by $\mathrm{C}$-section tend to be sicker and hence the choice of the mode of delivery. On the other hand, babies who are born by C-section may present with respiratory symptoms due to transient tachypnea of the newborn that could mimic clinical pneumonia. Within our cohort, infants who received more days of antibiotics required more days on ventilator, though this did not achieve statistical significance.

So far, there is no study that can define "healthy microbiota" in a preterm infant. Our study of microbiome established in preterm infants who have received only $2 \mathrm{~d}$ of antibiotic therapy is perhaps the healthiest cohort whose microbiome has been described. Previous studies have called for more judicious use of antibiotics given the known association between antibiotic use and NEC, but no causative mechanism has been provided. The changes in microbiota described in our current study may provide some insight in this association. Just as we have not defined a "healthy" microbiome, no one has defined the "NEC microbiome" either. The standard of care for NEC has been broad-spectrum antibiotic therapy and cessation of feeds. This seems to suggest that antibiotic treatment for NEC may eradicate the detrimental colonization and allow redevelopment of a more beneficial microbiota from a clean slate. There has been 


\section{Articles $\mid$ Dardaset al.}

a

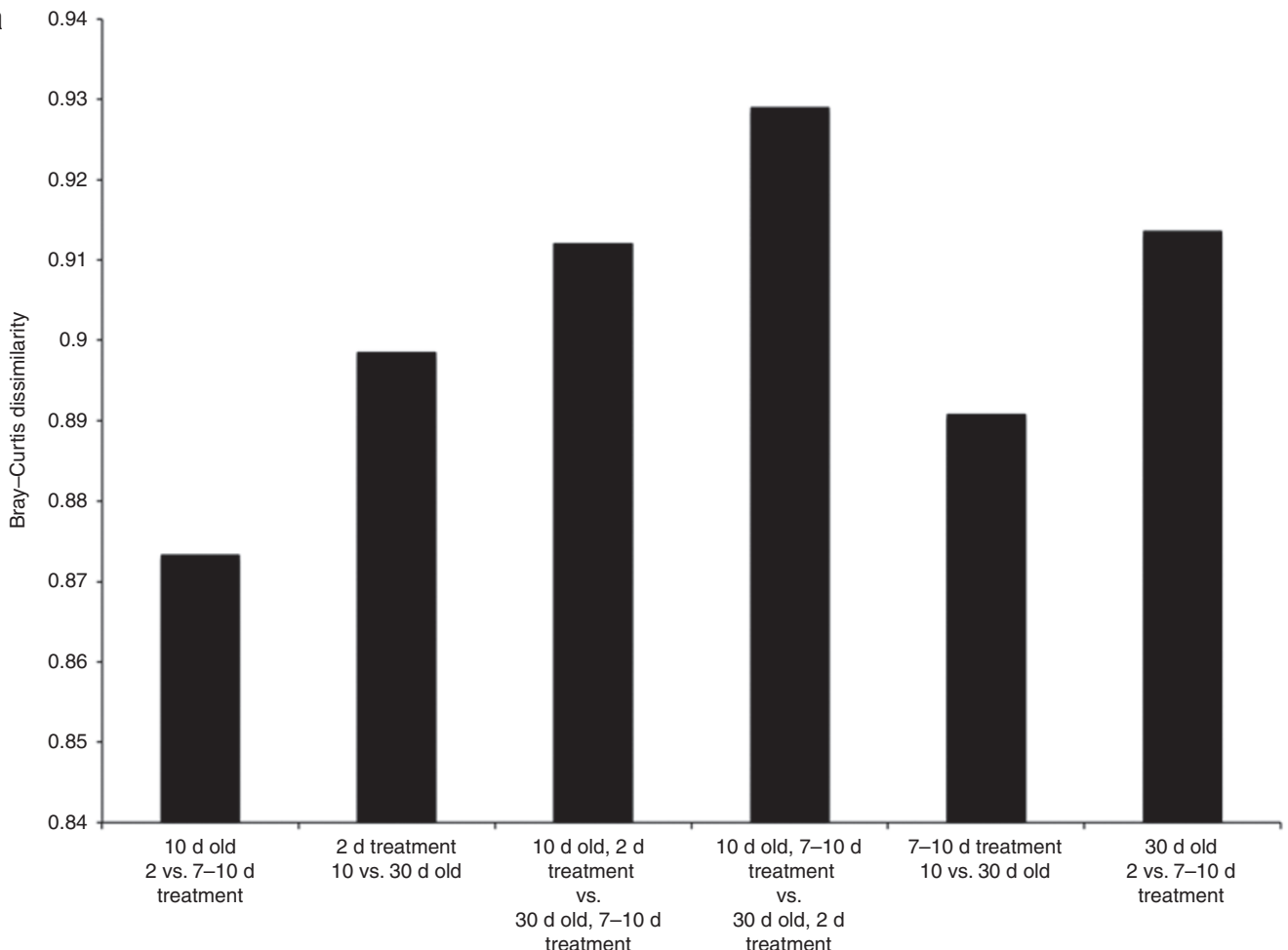

b

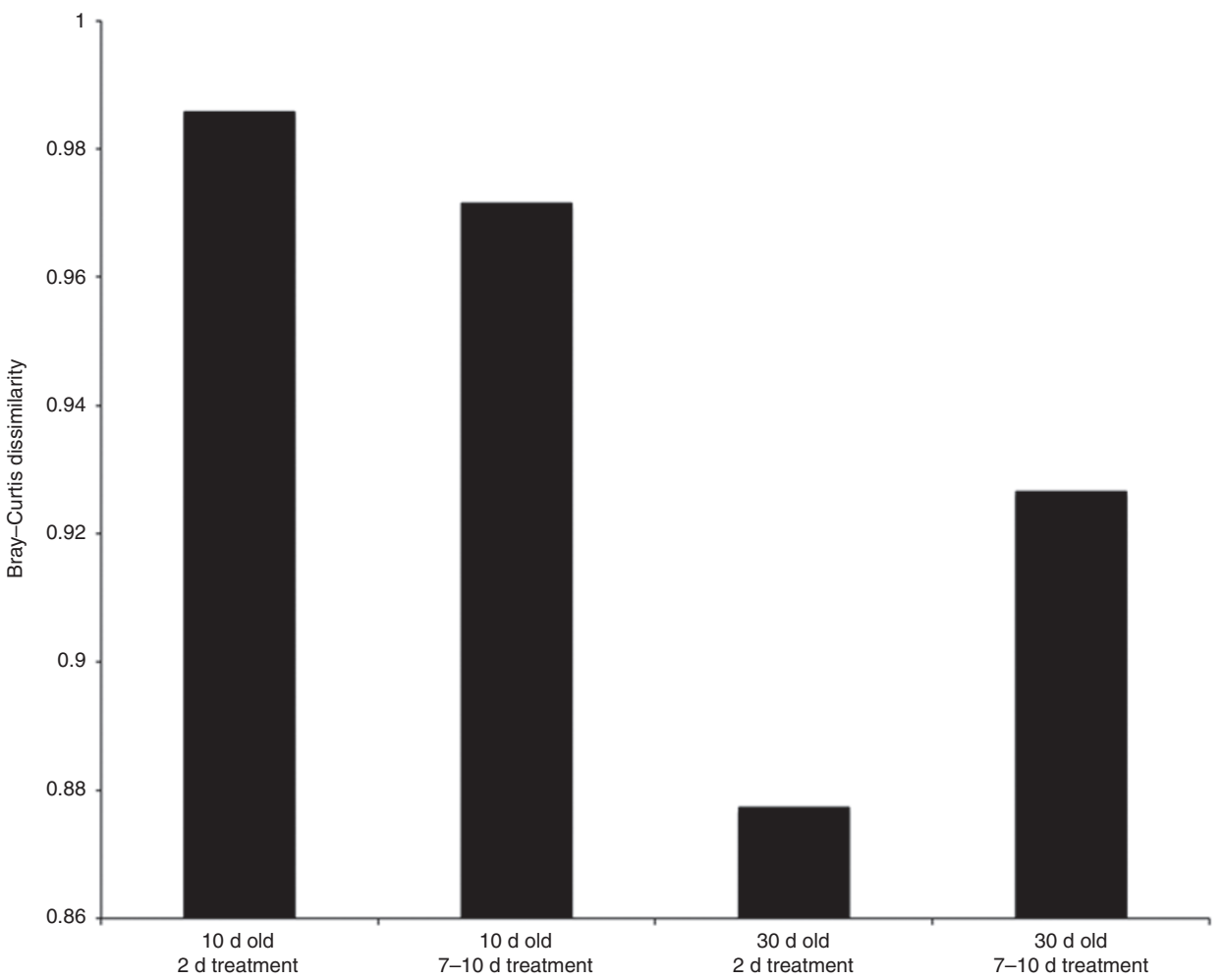

Figure 5. Bray-Curtis dissimilarity index ( $\beta$-diversity) based on age, antibiotic treatment, and dissimilarity between typical and atypical subgroups.

(a) Mean dissimilarity between all members of each group based on age and antibiotic treatment. (b) Mean dissimilarity between typical and atypical subgroups.

evidence of perturbation in distal gut microbiome caused by antibiotic therapy that lasts much longer than the 1-mo span for our current study (15). Our current study does not address the durability of any changes caused by antibiotic use during the newborn period or if there is any parallel in the pattern of microbiome establishment between newborn preterm infant and those whose microbiota has been previously established and then altered because of antibiotic therapy. While most 
studies have emphasized the relationship between neonatal intestinal microbiome and the onset of NEC, we must not lose sight of longer-term consequences of neonatal microbiome and its impact on the ontogeny of other biologic processes such as the development of the immune system. Our report does not provide sufficient evidence to suggest any alteration in clinical practice. Studies of much larger scale with much longer follow-up will be necessary to understand these issues. These questions will help direct future investigations.

\section{METHODS}

This study was performed at the University of Rochester Medical Center from 1 January to 31 December 2012. The study was approved by the Research Subject Review Boards at the University of Rochester. All admissions born at gestational age $<32 \mathrm{wk}$ were screened for eligibility irrespective of gender, race, or ethnic background. Eligible subjects were preterm infants born between $240 / 7$ and $316 / 7$ wk gestational age who received a cumulative total of $\geq 100 \mathrm{ml} / \mathrm{kg}$ of either $>50 \%$ breast milk or exclusive premature formula within the first 10 $\mathrm{d}$ of life. Exclusion criteria include NPO for $\geq 7 \mathrm{~d}$ during the study period, congenital abdominal wall defect or gastrointestinal anomalies, NEC, spontaneous intestinal perforation within the first $10 \mathrm{~d}$ of life, and a second course of antibiotic therapy within the first $10 \mathrm{~d}$ of life. The parents of identified eligible subjects were approached for consent to enroll in the study by day of life 7 .

Rectal swabs were used to collected fecal material from consented subjects at two time points, $10 \pm 1$ and $30 \pm 2 \mathrm{~d}$ of age. Each sampling involved inserting a sterile Copan flocked nylon swab (Copan Diagnostics, Murrieta, CA) moistened with normal saline beyond the sphincters into the rectum and then twirled. Each sample was immediately placed into a nucleic acid stabilizing reagent and stored at -80 ${ }^{\circ} \mathrm{C}$ until DNA extraction. All sampling swabs, plastic ware, buffers, and reagents used for collection of samples and extraction of nucleic acids were sterile and UV-irradiated to insure no contamination from sources outside of the infant and sample. Considerations were given to collect fecal samples from diapers. However, these infants do not necessarily have daily bowel movements. They have occasionally received glycerin suppositories to induce bowel movements, and the stool would have been contaminated by the suppositories. In addition, we were concerned about the sterility of the diapers from which we would collect samples and the potential for contamination from buttock skin flora.

Clinical care was at the discretion of treating physicians in terms of type and duration of antibiotic administration as well as the timing and volume of feeds. Feeding pattern (breast milk vs. formula) was also at discretion of mother and depended on breast milk availability. Medical records were reviewed to identify pertinent patient and maternal factors as well as feeding patterns. Percentage breast milk of total feeds was defined as the total breast milk volume of total volume of feeds calculated from birth up to the days of sample collection on days 10 and 30 .

Patient characteristics were assessed using a two-sample $t$-test for continuous variables and Fisher's exact test for categorical variables. Outcome variables were assessed using $t$-tests, and analyses of covariance were performed for confounders. Microbiome analysis of 10 and $30 \mathrm{~d}$ samples were performed to compare those who have received only $2 \mathrm{~d}$ of antibiotics and those who have received $\geq 7 \mathrm{~d}$ of antibiotics.

Total genomic DNA was extracted by a modified method using the Zymo Fecal DNA kit (Zymo Research, Irvine, CA) and FastPrep mechanical lysis (MPBio, Solon, OH). The V1-V3 region of bacterial 16S rRNA from each sample was amplified using 1 of 96 validated barcoded 454 primers. Two separate polymerase chain reaction amplifications for each sample were combined into a single sample and sequenced on a 454 GS Junior. One tube containing $10 \mu \mathrm{l}$ of sterile water was extracted and polymerase chain reaction amplified in parallel to detect possible contamination. Rarefaction curves were constructed from rectal and feeding samples at the beginning of the study to ensure that sequence depth was sufficient to determine the majority of bacterial diversity and identify rare bacteria.

For bacterial community data analysis, pyrosequence reads were first assessed for quality and then analyzed using phylogenetic and operational taxonomic unit methods in the Quantitative Insights into Microbial Ecology (QIIME) open source software, version 1.7 (16). QIIME was first used to demultiplex the barcoded reads and preprocess for quality. Reads were truncated if the average quality score in a 30 bp sliding window fell below 25 . Reads with more than six ambiguous base calls, homopolymers longer than six bp, errors in the primer sequence, more than one error in the barcode, or a length after quality trimming of less than $200 \mathrm{bp}$ were removed. Chimera detection and removal was performed de novo using UCHIME. To pick operational taxonomic units, USearch 5.2, a part of the QIIME package, was used to cluster the remaining reads based on percent identity, with a threshold of $97 \%$ in order to maximize taxonomic specificity. The May 2013 release of the GreenGenes reference database was used to provide seeds for all clusters, and clusters containing less than four reads were discarded. Taxonomic assignments were made to the resulting operational taxonomic units based on the seed of each cluster, using the Ribosomal Database Project classifier with the GreenGenes database of reference sequences and their known taxonomic assignments. Within each experimental condition, individuals were clustered based on constituent phyla using the hclust function of $\mathrm{R}$, which performs hierarchical clustering iteratively, using the Lance-Williams dissimilarity update formula and computing complete distances. The Shannon index was computed as a measure of $\alpha$-diversity for each individual, and the mean was taken for each group in each experimental condition. The Bray-Curtis dissimilarity index between every pair of individuals was calculated as a measure of $\beta$-diversity. The average dissimilarity between every individual in each pair of experimental conditions was calculated, as was the average dissimilarity between every member of each pair of subgroups within each experimental condition.

\section{ACKNOWLEDGMENTS}

The authors thank Brooke Hamilton, Laura Nally, and Bianca Redhead, for their valuable work in sample processing. The authors also express their great appreciation to Patricia Chess and Rita Dadiz for their valuable and constructive suggestions during the planning and development of this research work. Acknowledgments extend to Hongyue Wang for her help with statistical analysis.

\section{STATEMENT OF FINANCIAL SUPPORT}

This project was funded by a grant from the Strong Children's Research Center University of Rochester Medical Center, Rochester, NY.

Disclosure: The authors do not have any financial ties to products in the study or potential/perceived conflicts of interest

\section{REFERENCES}

1. Martin R, Nauta AJ, Ben Amor K, Knippels LM, Knol J, Garssen J. Early life: gut microbiota and immune development in infancy. Benef Microbes 2010;1:367-82.

2. Wang Y, Hoenig JD, Malin KJ, et al. 16S rRNA gene-based analysis of fecal microbiota from preterm infants with and without necrotizing enterocolitis. ISME J 2009;3:944-54.

3. Torrazza RM, Neu J. The altered gut microbiome and necrotizing enterocolitis. Clinics in Perinatology 2013;40:93-108.

4. de la Cochetiere MF, Piloquet H, des Robert C, Darmaun D, Galmiche JP, Roze JC. Early intestinal bacterial colonization and necrotizing enterocolitis in premature infants: the putative role of Clostridium. Pediatr Res 2004;56:366-70.

5. Mai V, Young CM, Ukhanova $\mathrm{M}$, et al. Fecal microbiota in premature infants prior to necrotizing enterocolitis. PLoS One 2011;6:e20647.

6. Guillet R, Stoll BJ, Cotten CM, et al. Association of H2-blocker therapy and higher incidence of necrotizing enterocolitis in very low birth weight infants. Pediatrics 2006;117:e137-42.

7. Cotten CM, Taylor S, Stoll B, et al. Prolonged duration of initial empirical antibiotic treatment is associated with increased rates of necrotizing 
enterocolitis and death for extremely low birth weight infants. Pediatrics 2009;123:58-66.

8. Lucas A, Cole TJ. Breast milk and neonatal necrotising enterocolitis. Lancet 1990;336:1519-23.

9. Quigley MA, Henderson G, Anthony MY, McGuire W. Formula milk versus donor breast milk for feeding preterm or low birth weight infants. The Cochrane Database of Systematic Reviews 2007:CD002971.

10. Deshpande G, Rao S, Patole S. Probiotics for prevention of necrotising enterocolitis in preterm neonates with very low birthweight: a systematic review of randomised controlled trials. Lancet 2007;369:1614-20.

11. Lin $\mathrm{HC}, \mathrm{Hsu} \mathrm{CH}$, Chen $\mathrm{HL}$, et al. Oral probiotics prevent necrotizing enterocolitis in very low birth weight preterm infants: a multicenter, randomized, controlled trial. Pediatrics 2008;122:693-700.
12. Alfaleh K, Anabrees J, Bassler D. Probiotics reduce the risk of necrotizing enterocolitis in preterm infants: a meta-analysis. Neonatology 2010;97:93-9.

13. Koenig JE, Spor A, Scalfone N, et al. Succession of microbial consortia in the developing infant gut microbiome. Proc Natl Acad Sci USA 2011;108:Suppl 1:4578-85.

14. Normann E, Fahlén A, Engstrand L, Lilja HE. Intestinal microbial profiles in extremely preterm infants with and without necrotizing enterocolitis. Acta Paediatr 2013;102:129-36.

15. Dethlefsen L, Relman DA. Incomplete recovery and individualized responses of the human distal gut microbiota to repeated antibiotic perturbation. Proc Natl Acad Sci USA 2011;108:Suppl 1:4554-61.

16. Caporaso JG, Kuczynski J, Stombaugh J, et al. QIIME allows analysis of high-throughput community sequencing data. Nat Methods 2010;7:335-6. 